MATHEMATICS OF COMPUTATION

Volume 67, Number 224, October 1998, Pages 1591-1599

S 0025-5718(98)00978-8

\title{
FACTORIZING COMPLEX SYMMETRIC MATRICES WITH POSITIVE DEFINITE REAL AND IMAGINARY PARTS
}

\author{
NICHOLAS J. HIGHAM
}

\begin{abstract}
Complex symmetric matrices whose real and imaginary parts are positive definite are shown to have a growth factor bounded by 2 for LU factorization. This result adds to the classes of matrix for which it is known to be safe not to pivot in LU factorization. Block $\mathrm{LDL}^{\mathrm{T}}$ factorization with the pivoting strategy of Bunch and Kaufman is also considered, and it is shown that for such matrices only $1 \times 1$ pivots are used and the same growth factor bound of 2 holds, but that interchanges that destroy band structure may be made. The latter results hold whether the pivoting strategy uses the usual absolute value or the modification employed in LINPACK and LAPACK.
\end{abstract}

\section{INTRODUCTION}

There are three main classes of matrix for which it is known to be safe not to pivot when computing an LU factorization: matrices diagonally dominant by rows or columns, Hermitian positive definite matrices, and totally nonnegative matrices. Here, we identify another class of matrices with this highly desirable property: complex symmetric matrices whose real and imaginary parts are both positive definite. Complex symmetric matrices arise frequently, particularly in algebraic eigenvalue problems [16], [17], and numerical methods that exploit their structure can be developed; see, for example, [3], [7], [20]. Complex symmetric matrices with positive definite real and imaginary parts arise in calculations with Padé approximations to the exponential [6]; in this application the matrices are banded.

In Section 2 we show that a complex symmetric matrix with positive definite real and imaginary parts can be factorized by LU factorization without pivoting and that the growth factor is bounded by 2 . In Section 3 we discuss how best to solve a linear system with such a coefficient matrix. This leads to consideration in Section 4 of block LDL ${ }^{\mathrm{T}}$ factorization using the Bunch-Kaufman pivoting strategy, which we find uses only $1 \times 1$ pivots and leads to a growth factor bound of 2 , but may make interchanges that destroy band structure.

\section{Complex SymmetriC POSITIVE DEFinite MATRICES}

We call any matrix of the form

$A=B+i C, \quad B \in \mathbb{R}^{n \times n}$ and $C \in \mathbb{R}^{n \times n}$ both symmetric positive definite,

Received by the editor December 8, 1996.

1991 Mathematics Subject Classification. Primary 65F05.

Key words and phrases. Complex symmetric matrices, LU factorization, diagonal pivoting factorization, block $\mathrm{LDL}^{\mathrm{T}}$ factorization, Bunch-Kaufman pivoting strategy, growth factor, band matrix, LINPACK, LAPACK. 
a CSPD matrix (standing for complex symmetric positive definite). Note that if $A$ is a CSPD matrix then $\alpha A$ is not, in general, for $\alpha \in \mathbb{C}$. It is possible that in some applications CSPD matrices arise in scaled form and their structure goes unrecognised.

We begin by establishing some basic facts about CSPD matrices.

Lemma 2.1. A CSPD matrix is nonsingular.

Proof. Let $B+i C$ be a CSPD matrix and consider the linear system $(B+i C)(x+i y)$ $=d+i e$. It may be rewritten as

$$
\left[\begin{array}{cc}
B & -C \\
C & B
\end{array}\right]\left[\begin{array}{l}
x \\
y
\end{array}\right]=\left[\begin{array}{l}
d \\
e
\end{array}\right]
$$

The matrix of this linear system is nonsymmetric positive definite ${ }^{1}$ and therefore nonsingular. Hence $d+i e=0$ implies $x+i y=0$, showing that $B+i C$ is nonsingular.

Lemma 2.2. A CSPD matrix $A$ has a unique $L U$ factorization $A=L U$.

Proof. We have to show that the leading principal submatrices of $A$ are nonsingular (see, for example, [12, Theorem 9.1]). But any principal submatrix of a CSPD matrix is CSPD and so nonsingular.

Lemma 2.2 shows that an LU factorization exists without the need for pivoting, but it does not address the stability of such a factorization. The standard backward error analysis of Wilkinson shows that if an LU factorization without pivoting produces a computed solution $\widehat{x}$ to $A x=b$, where $A \in \mathbb{C}^{n \times n}$, then

$$
(A+\Delta A) \widehat{x}=b, \quad\|\Delta A\|_{\infty} \leq c n^{3} \rho_{n} u\|A\|_{\infty}+O\left(u^{2}\right),
$$

where $c$ is a modest constant and $u$ is the unit roundoff (see, for example, [12, Theorem 9.5, Problem 9.8]). The quantity $\rho_{n}$ is the growth factor, defined by

$$
\rho_{n}=\frac{\max _{i, j, k}\left|a_{i j}^{(k)}\right|}{\max _{i, j}\left|a_{i j}\right|}
$$

where the $a_{i j}^{(k)}$ are the elements of the Schur complements arising during the factorization. Hence backward stability is guaranteed if $\rho_{n}$ is small. It is a nontrivial task to bound $\rho_{n}$ for CSPD matrices because the multipliers can be arbitrarily large, just as for real symmetric positive definite matrices. As a simple example, for the CSPD matrix

$$
A=(1+i)\left[\begin{array}{cc}
\epsilon^{2} & \epsilon \\
\epsilon & 2
\end{array}\right], \quad \epsilon>0
$$

the first multiplier in Gaussian elimination is $\epsilon^{-1}$. In the rest of this section we show that a very satisfactory growth factor bound can be obtained.

First, we show that Schur complements inherit the CSPD structure.

Lemma 2.3. If

$$
A=\underset{n-p}{p}\left[\begin{array}{cc}
p & n-p \\
A_{11} & A_{21}^{T} \\
A_{21} & A_{22}
\end{array}\right]
$$

${ }^{1} \mathrm{~A}$ matrix $E \in \mathbb{R}^{n \times n}$ is (nonsymmetric) positive definite if $x^{T} E x>0$ for all $0 \neq x \in \mathbb{R}^{n}$ or, equivalently, if the symmetric part $\left(E+E^{T}\right) / 2$ is positive definite. 
is a CSPD matrix, then so is $S=A_{22}-A_{21} A_{11}^{-1} A_{21}^{T}$, the Schur complement of $A_{11}$ in $A$.

Proof. Clearly $S$ is symmetric; we have to show that its real and imaginary parts are positive definite. Write $A=B+i C$ with $B$ and $C$ partitioned conformally with A. Then

$$
S=B_{22}+i C_{22}-\left(B_{21}+i C_{21}\right)(X+i Y)
$$

where

$$
\left(B_{11}+i C_{11}\right)(X+i Y)=B_{21}^{T}+i C_{21}^{T}
$$

Hence

$$
\left[\begin{array}{cc}
B_{11} & C_{11} \\
C_{11} & -B_{11}
\end{array}\right]\left[\begin{array}{c}
X \\
-Y
\end{array}\right]=\left[\begin{array}{l}
B_{21}^{T} \\
C_{21}^{T}
\end{array}\right]
$$

Thus

$$
\begin{aligned}
\operatorname{Re}(S) & =B_{22}-\left[\begin{array}{ll}
B_{21} & C_{21}
\end{array}\right]\left[\begin{array}{c}
X \\
-Y
\end{array}\right] \\
& =B_{22}-\left[\begin{array}{ll}
B_{21} & C_{21}
\end{array}\right]\left[\begin{array}{cc}
B_{11} & C_{11} \\
C_{11} & -B_{11}
\end{array}\right]^{-1}\left[\begin{array}{l}
B_{21}^{T} \\
C_{21}^{T}
\end{array}\right],
\end{aligned}
$$

which is the Schur complement of $\left[\begin{array}{cc}B_{11} & C_{11} \\ C_{11} & -B_{11}\end{array}\right]$ in

$$
G_{1}:=\left[\begin{array}{ccc}
B_{11} & C_{11} & B_{21}^{T} \\
C_{11} & -B_{11} & C_{21}^{T} \\
B_{21} & C_{21} & B_{22}
\end{array}\right]=\Pi\left[\begin{array}{ccc}
B_{11} & B_{21}^{T} & C_{11} \\
B_{21} & B_{22} & C_{21} \\
C_{11} & C_{21}^{T} & -B_{11}
\end{array}\right] \Pi^{T}=: \Pi G_{2} \Pi^{T},
$$

for a permutation matrix $\Pi$. Recall that the inertia of a symmetric matrix is an ordered triple $\left(i_{+}, i_{-}, i_{0}\right)$, where $i_{+}, i_{-}$, and $i_{0}$ are, respectively, the number of positive, negative, and zero eigenvalues. We now use the fact that for a real, symmetric matrix $H$, if a leading principal submatrix $H_{11}$ is nonsingular, then $\operatorname{inertia}(H)=\operatorname{inertia}\left(H_{11}\right)+\operatorname{inertia}\left(H / H_{11}\right)$, where $H / H_{11}$ denotes the Schur complement of $H_{11}$ in $H$ (see, for example, [10]). We obtain

$$
\begin{aligned}
\operatorname{inertia}\left(G_{2}\right) & =\operatorname{inertia}(B)+\operatorname{inertia}\left(-B_{11}-\left[\begin{array}{ll}
C_{11} & C_{21}^{T}
\end{array}\right] B^{-1}\left[\begin{array}{l}
C_{11} \\
C_{21}
\end{array}\right]\right) \\
& =(n, p, 0), \\
\operatorname{inertia}\left(G_{1}\right) & =\operatorname{inertia}\left(\left[\begin{array}{cc}
B_{11} & C_{11} \\
C_{11} & -B_{11}
\end{array}\right]\right)+\operatorname{inertia}(\operatorname{Re}(S)) \\
& =(p, p, 0)+\operatorname{inertia}(\operatorname{Re}(S)) .
\end{aligned}
$$

But $G_{1}$ and $G_{2}$ have the same inertia, so inertia $(\operatorname{Re}(S))=(n-p, 0,0)$, as required. The positive definiteness of $\operatorname{Im}(S)$ is proved similarly.

The next result implies that the largest element of a CSPD matrix lies on the diagonal.

Lemma 2.4. If $A$ is a CSPD matrix, then

$$
\left|a_{i j}\right|<\sqrt{\left|a_{i i}\right|\left|a_{j j}\right|}
$$


Proof. Write $A=B+i C$. Using the well-known fact that (2.2) is true for a real symmetric positive definite matrix we have

$$
\begin{aligned}
\left|a_{i j}\right| & =\sqrt{b_{i j}^{2}+c_{i j}^{2}} \\
& <\sqrt{b_{i i} b_{j j}+c_{i i} c_{j j}} \\
& <\sqrt{\sqrt{b_{i i}^{2}+c_{i i}^{2}} \sqrt{b_{j j}^{2}+c_{j j}^{2}}} \quad \text { (Cauchy-Schwarz inequality) } \\
& =\sqrt{\left|a_{i i}\right|\left|a_{j j}\right|} .
\end{aligned}
$$

Theorem 2.5. The growth factor for a CSPD matrix $A \in \mathbb{C}^{n \times n}$ satisfies $\rho_{n}<2$ and this bound is the best possible.

Proof. In the notation of Lemma 2.3, we have to show that every element of the Schur complement matrix $S$ satisfies $\left|s_{i j}\right|<2 \max _{i, j}\left|a_{i j}\right|$. Since $S$ is CSPD by Lemma 2.3, we have

$$
\operatorname{Re}\left(\left(A_{21} A_{11}^{-1} A_{21}^{T}\right)_{j j}\right)<\operatorname{Re}\left(\left(A_{22}\right)_{j j}\right), \quad \operatorname{Im}\left(\left(A_{21} A_{11}^{-1} A_{21}^{T}\right)_{j j}\right)<\operatorname{Im}\left(\left(A_{22}\right)_{j j}\right) .
$$

Squaring, adding and then taking the square root gives

$$
\left|\left(A_{21} A_{11}^{-1} A_{21}^{T}\right)_{j j}\right|<\left|\left(A_{22}\right)_{j j}\right| .
$$

Thus $\left|s_{j j}\right|<2\left|\left(A_{22}\right)_{j j}\right|<2 \max _{i, j}\left|a_{i j}\right|$. Hence, by Lemma 2.4,

$$
\left|s_{i j}\right|<\sqrt{\left|s_{i i}\right|\left|s_{j j}\right|}<2 \max _{i, j}\left|a_{i j}\right| .
$$

Finally, consider the LU factorization

$$
A=\left[\begin{array}{cc}
1+i & 1-i \\
1-i & 1+i
\end{array}\right]=\left[\begin{array}{cc}
1 & 0 \\
-i & 1
\end{array}\right]\left[\begin{array}{cc}
1+i & 1-i \\
0 & 2(1+i)
\end{array}\right]
$$

for which $\rho_{2}=2$. The matrix $A$ is not CSPD, because its real and imaginary parts are symmetric positive semidefinite and singular. But $A+\epsilon(1+i) I$ is CSPD for $\epsilon>0$ and as $\epsilon \rightarrow 0, \rho_{2} \rightarrow 2$. Hence $\rho_{n}<2$ is the best possible bound.

We conclude from Theorem 2.5 that LU factorization without pivoting is normwise backward stable when applied to CSPD systems.

\section{Solving CPSD systems}

How should we solve a CSPD system $A x=b$ ? The natural choice is LU factorization without pivoting, which we have proved to be normwise backward stable. Symmetry can be exploited in the implementation, making the cost $n^{3} / 3$ complex operations. Since no pivoting is used, any band structure in $A$ is preserved in the factorization. 
An alternative to working in complex arithmetic is to solve an equivalent real system. In preference to the real system (2.1), we consider the symmetric modification

$$
\left[\begin{array}{cc}
B & C \\
C & -B
\end{array}\right]\left[\begin{array}{c}
x \\
-y
\end{array}\right]=\left[\begin{array}{l}
d \\
e
\end{array}\right]
$$

The coefficient matrix is a special case of a symmetric quasidefinite matrix [19]. For such a matrix an $\mathrm{LDL}^{\mathrm{T}}$ factorization exists. Although $\mathrm{LDL}^{\mathrm{T}}$ without pivoting is not always stable for symmetric quasidefinite matrices, conditions under which stability is guaranteed are derived in [8], via corresponding analysis for nonsymmetric positive definite real systems [9], [15]. For the system (3.1) we find that stability is assured if $\left\|C B^{-1} C\right\|_{2} /\|A\|_{2}$ is not too large.

Solving (3.1) requires $8 n^{3} / 3$ real operations as opposed to $n^{3} / 3$ complex operations to solve the original complex system directly, and it requires the same amount of storage. A complex operation requires between 2 and 8 real operations, so solving the complex system should, in principal, be the more efficient option, but the actual relative costs of real and complex arithmetic will depend on the computing environment.

LINPACK [4] and LAPACK [1] do not contain routines for LU factorization without pivoting applied to complex symmetric matrices. The user who does not wish to write special-purpose code but wishes to take advantage of symmetry would therefore have to treat the system as a general complex symmetric system and solve it using block $\mathrm{LDL}^{\mathrm{T}}$ factorization-based routines: LINPACK's ${ }^{2}$ CSIFA, CSISL, or LAPACK's CSYTRF, CSYTRS. These routines use the pivoting strategy of Bunch and Kaufman [2]. Two important questions arise. When the Bunch-Kaufman pivoting strategy is applied to a CSPD system

1. Is the growth factor bound $\rho_{n}<2$ still valid?

2. Is bandwidth preserved for a banded matrix? (Implementations of block $\mathrm{LDL}^{\mathrm{T}}$ factorization with the Bunch-Kaufman pivoting strategy for banded real symmetric matrices are given by Jones and Patrick [13], [14].)

These questions are answered in the next section.

\section{BLOCK LDL ${ }^{T}$ FACTORIZATION}

We briefly summarize block $\mathrm{LDL}^{\mathrm{T}}$ factorization for a complex symmetric matrix.

Let $A \in \mathbb{C}^{n \times n}$ be complex symmetric. If $A$ is nonzero then there is a permutation $\Pi$ and an integer $s=1$ or 2 so that

$$
\Pi A \Pi^{T}={ }_{n-s}^{s}\left[\begin{array}{cc}
s & n-s \\
C & B
\end{array}\right],
$$

with $E$ nonsingular. Then we have the factorization

$$
\Pi A \Pi^{T}=\left[\begin{array}{cc}
I_{s} & 0 \\
C E^{-1} & I_{n-s}
\end{array}\right]\left[\begin{array}{cc}
E & 0 \\
0 & B-C E^{-1} C^{T}
\end{array}\right]\left[\begin{array}{cc}
I_{s} & E^{-1} C^{T} \\
0 & I_{n-s}
\end{array}\right] .
$$

\footnotetext{
${ }^{2}$ LINPACK and LAPACK support block LDL ${ }^{T}$ factorization for three different types of matrix: real symmetric, complex symmetric, and complex Hermitian.
} 
Repetition of this process on the Schur complement $S=B-C E^{-1} C^{T}$ leads to a block $\mathrm{LDL}^{\mathrm{T}}$ factorization

$$
P A P^{T}=L D L^{T},
$$

where $L$ is unit lower triangular and $D$ is block diagonal with each diagonal block having dimension 1 or 2. To describe the pivoting strategy of Bunch and Kaufman [2] it suffices to describe the choice of $\Pi$ and $s$ on the first stage of the factorization.

Algorithm 4.1 (Bunch-Kaufman pivoting strategy). This algorithm determines the pivot for the first stage of block $L D L^{T}$ factorization with the Bunch-Kaufman pivoting strategy applied to a complex symmetric matrix $A \in \mathbb{C}^{n \times n}$.

$\alpha:=(1+\sqrt{17}) / 8(\approx 0.64)$

$\lambda:=\|A(2: n, 1)\|_{\infty}$

If $\lambda=0$ there is nothing to do on this stage of the elimination.

$r:=\min \left\{i \geq 2:\left|a_{i 1}\right|=\lambda\right\}$

if $\left|a_{11}\right| \geq \alpha \lambda$

(1) use $a_{11}$ as a $1 \times 1$ pivot $(s=1, \Pi=I)$.

else

$$
\sigma:=\left\|\left[\begin{array}{l}
A(1: r-1, r) \\
A(r+1: n, r)
\end{array}\right]\right\|_{\infty}
$$

if $\left|a_{11}\right| \sigma \geq \alpha \lambda^{2}$

use $a_{11}$ as a $1 \times 1$ pivot $(s=1, \Pi=I)$.

else if $\left|a_{r r}\right| \geq \alpha \sigma$

use $a_{r r}$ as a $1 \times 1$ pivot $(s=1, \Pi$ swaps rows and columns

1 and $r$ ).

else

use $\left[\begin{array}{ll}a_{11} & a_{r 1} \\ a_{r 1} & a_{r r}\end{array}\right]$ as a $2 \times 2$ pivot $(s=2, \Pi$ swaps rows and

columns 2 and $r$ ).

end

end

LINPACK and LAPACK eschew the usual complex absolute value $|x+i y|=$ $\sqrt{x^{2}+y^{2}}$. Instead they use

$$
|x+i y|:=|x|+|y|
$$

which is less expensive to evaluate and less prone to overflow and underflow in floating point arithmetic. The usual absolute value is used in the routine diagpiv in the MatLaB Test Matrix Toolbox [11]. We will consider both choices of absolute value in Algorithm 4.1. (We assume that the $\infty$-norm utilizes whichever absolute value has been chosen). We write

$$
\begin{aligned}
\operatorname{abs}(x+i y) & :=\sqrt{x^{2}+y^{2}}, \\
\operatorname{abs}_{1}(x+i y) & :=|x|+|y|,
\end{aligned}
$$

and we write $|z|$ only when $z$ is real or when we wish to make statements holding for both choices of absolute value.

We wish to determine whether Algorithm 4.1 simplifies for a CSPD matrix. Serbin [18] considered this question and claimed, incorrectly, that for the absolute value abs 1 no pivoting is required, that is, that Algorithm 4.1 leads to $P=I$ in 
(4.2). His argument was based on the erroneous assumption that the largest element in any column of a real, symmetric positive definite matrix lies on the diagonal. The following lemma summarizes the possible behavior of Algorithm 4.1 for both choices of absolute value.

Lemma 4.2. Consider the pivoting strategy of Algorithm 4.1 for either choice of absolute value. For a CSPD matrix no $2 \times 2$ pivots are chosen. However, each of the cases (1), (2) and (3) that chooses a $1 \times 1$ pivot may be selected.

Proof. The conditions that must hold for a $2 \times 2$ pivot

$$
E=\left[\begin{array}{ll}
a_{11} & a_{r 1} \\
a_{r 1} & a_{r r}
\end{array}\right]=: F+i G, \quad\left|a_{r 1}\right|=\lambda
$$

to be selected on the first stage of the factorization are as follows:

$$
\begin{aligned}
\left|a_{11}\right| & <\alpha \lambda, \\
\left|a_{11}\right| \sigma & <\alpha \lambda^{2}, \\
\left|a_{r r}\right| & <\alpha \sigma, \\
\left|a_{11}\right|\left|a_{r r}\right| & <\alpha^{2} \lambda^{2},
\end{aligned}
$$

where the fourth inequality is a consequence of the previous two (note that (4.4c) implies $\sigma \neq 0$ ). Since $A$ is CSPD, $E$ is CSPD and so $F$ and $G$ are symmetric positive definite.

Consider, first, the absolute value abs. From $\operatorname{det}(F)=f_{11} f_{22}-f_{21}^{2}>0$ and $\operatorname{det}(G)>0$ we have the inequalities

$$
f_{11}^{2} f_{22}^{2}>f_{21}^{4}, \quad g_{11}^{2} g_{22}^{2}>g_{21}^{4}, \quad \frac{f_{11}^{2} g_{22}^{2}+g_{11}^{2} f_{22}^{2}}{2} \geq f_{11} g_{22} g_{11} f_{22}>f_{21}^{2} g_{21}^{2},
$$

which together imply

$$
\left(f_{11}^{2}+g_{11}^{2}\right)\left(f_{22}^{2}+g_{22}^{2}\right)>\left(f_{21}^{2}+g_{21}^{2}\right)^{2} .
$$

Since

$$
\operatorname{abs}(E)=\left[\begin{array}{ll}
\sqrt{f_{11}^{2}+g_{11}^{2}} & \sqrt{f_{21}^{2}+g_{21}^{2}} \\
\sqrt{f_{21}^{2}+g_{21}^{2}} & \sqrt{f_{22}^{2}+g_{22}^{2}}
\end{array}\right]=\left[\begin{array}{cc}
\operatorname{abs}\left(a_{11}\right) & \lambda \\
\lambda & \operatorname{abs}\left(a_{r r}\right)
\end{array}\right]
$$

it follows that

$$
\operatorname{abs}\left(a_{11}\right) \operatorname{abs}\left(a_{r r}\right)>\lambda^{2} \geq \alpha^{2} \lambda^{2},
$$

which shows that $(4.4 \mathrm{~d})$ cannot hold.

Turning to the absolute value abs ${ }_{1}$, we have

$$
\begin{aligned}
\operatorname{abs}_{1}\left(a_{11}\right) \operatorname{abs}_{1}\left(a_{r r}\right)-\operatorname{abs}_{1}\left(a_{r 1}\right)^{2} & =\left(f_{11}+g_{11}\right)\left(f_{22}+g_{22}\right)-\left(\left|f_{21}\right|+\left|g_{21}\right|\right)^{2} \\
& =\operatorname{det}(|F|+|G|) \\
& >0
\end{aligned}
$$

where we have used the facts that if $E \in \mathbb{R}^{2 \times 2}$ is symmetric positive definite then so is $|E|$ and that the sum of two symmetric positive definite matrices is symmetric positive definite. Hence

$$
\operatorname{abs}_{1}\left(a_{11}\right) \operatorname{abs}_{1}\left(a_{r r}\right)>\lambda^{2} \geq \alpha^{2} \lambda^{2},
$$

which shows, once again, that $(4.4 \mathrm{~d})$ cannot hold. 
We have shown that for both choices of absolute value a $2 \times 2$ pivot is not selected on the first stage of the factorization. In view of Lemma 2.3, the same argument applies to each stage of the factorization.

It remains to show that cases (1), (2) and (3) in Algorithm 4.1 can all be selected. We give examples for real, symmetric positive definite matrices $A$, from which corresponding CSPD examples can be constructed for either absolute value by taking $A+i \epsilon I$ for suitably small $\epsilon>0$. Case (1) is selected for $A=I$. Case (2) is selected for

$$
A=\left[\begin{array}{ccc}
\frac{3 \alpha}{4} & 1 & 0 \\
1 & 4 & 2 \\
0 & 2 & 4
\end{array}\right]
$$

Case (3) is selected for

$$
A=\left[\begin{array}{cc}
1 & \theta \\
\theta & 2 \theta^{2}
\end{array}\right], \quad \theta>\alpha^{-1}
$$

We can now draw some conclusions, all of which hold for either choice of absolute value. Lemma 4.2 shows that only $1 \times 1$ pivots are used by the Bunch-Kaufman pivoting strategy when applied to a CSPD matrix, so that $D$ is diagonal, but that interchanges may nevertheless be performed. We see that block $\mathrm{LDL}^{\mathrm{T}}$ factorization therefore essentially produces the LU factorization of a permuted matrix $P A P^{T}$, with $D L^{T}$ equal to the $U$ factor, because of the uniqueness of the LU factorization shown in Lemma 2.2. Since $P A P^{T}$ is a CSPD matrix if $A$ is, it follows that the growth factor bound $\rho_{n}<2$ holds; this bound is much stronger than the bound $^{3} \rho_{n} \leq(2.57)^{n-1}$ available for the Bunch-Kaufman pivoting strategy applied to general complex symmetric matrices [2].

We can also conclude that when the Bunch-Kaufman pivoting strategy is used with a banded CSPD matrix, the bandwidth may not be preserved, and it is easy to generate illustrative examples empirically.

Finally, we mention that Sorensen and Van Loan [5, Section 5.3.2] suggest modifying Algorithm 4.1 by redefining $\sigma:=\|A(:, r)\|_{\infty}$. Their reasoning is that this modification does not affect any of the main properties of the algorithm (the growth factor bound and error analysis remain unchanged, for example), but for (real) symmetric positive definite matrices the modified strategy results in no interchanges being made. It is easy to show that, with this modification, no interchanges are made for CSPD matrices either.

\section{REFERENCES}

[1] E. Anderson, Z. Bai, C. H. Bischof, J. W. Demmel, J. J. Dongarra, J. J. Du Croz, A. Greenbaum, S. J. Hammarling, A. McKenney, S. Ostrouchov, and D. C. Sorensen, LAPACK users' guide, Release 2.0, second ed., Society for Industrial and Applied Mathematics, Philadelphia, PA, USA, 1995.

[2] James R. Bunch and Linda Kaufman, Some stable methods for calculating inertia and solving symmetric linear systems, Math. Comp. 31 (1977), no. 137, 163-179. MR 55:1714

\footnotetext{
${ }^{3}$ In fact, the proofs of the bound $\rho_{n} \leq(2.57)^{n-1}$ in [2], [12, Section 10.4] are valid for the absolute value abs only. For abs 1 , as used in LINPACK and LAPACK, it is not clear what is the best bound for the growth factor.
} 
[3] Jane K. Cullum and Ralph A. Willoughby, A QL procedure for computing the eigenvalues of complex symmetric tridiagonal matrices, SIAM J. Matrix Anal. Appl. 17 (1996), no. 1, 83-109. MR 96k:65028

[4] J. J. Dongarra, J. R. Bunch, C. B. Moler, and G. W. Stewart, LINPACK users' guide, Society for Industrial and Applied Mathematics, Philadelphia, PA, USA, 1979.

[5] Jack J. Dongarra, Iain S. Duff, Danny C. Sorensen, and Henk A. van der Vorst, Solving linear systems on vector and shared memory computers, Society for Industrial and Applied Mathematics, Philadelphia, PA, USA, 1991. MR 92a:65001

[6] Graeme Fairweather, A note on the efficient implementation of certain Padé methods for linear parabolic problems, BIT 18 (1978), 106-109. MR 58:8326

[7] Roland W. Freund, Conjugate gradient-type methods for linear systems with complex symmetric coefficient matrices, SIAM J. Sci. Stat. Comput. 13 (1992), no. 1, 425-448. MR 92i: 65066

[8] Philip E. Gill, Michael A. Saunders, and Joseph R. Shinnerl, On the stability of Cholesky factorization for symmetric quasidefinite systems, SIAM J. Matrix Anal. Appl. 17 (1996), no. 1, 35-46. MR 97a:15027

[9] Gene H. Golub and Charles F. Van Loan, Unsymmetric positive definite linear systems, Linear Algebra and Appl. 28 (1979), 85-97. MR 80k:15016

[10] Emilie V. Haynsworth, Determination of the inertia of a partitioned matrix, Linear Algebra and Appl. 1 (1968), 73-81. MR 36:6440

[11] Nicholas J. Higham, The Test Matrix Toolbox for Matlab (version 3.0), Numerical Analysis Report No. 276, Manchester Centre for Computational Mathematics, Manchester, England, September 1995.

[12] _ Accuracy and stability of numerical algorithms, Society for Industrial and Applied Mathematics, Philadelphia, PA, USA, 1996. MR 97a:65047

[13] Mark T. Jones and Merrell L. Patrick, Bunch-Kaufman factorization for real symmetric indefinite banded matrices, SIAM J. Matrix Anal. Appl. 14 (1993), no. 2, 553-559. CMP 93:10

[14] _ Factoring symmetric indefinite matrices on high-performance architectures, SIAM J. Matrix Anal. Appl. 15 (1994), no. 1, 273-283. MR 94j:65040

[15] Roy Mathias, Matrices with positive definite Hermitian part: Inequalities and linear systems, SIAM J. Matrix Anal. Appl. 13 (1992), no. 2, 640-654. MR 92k:15041

[16] Giorgio Moro and Jack H. Freed, Calculation of ESR spectra and related Fokker-Planck forms by the use of the Lanczos algorithm, J. Chem. Phys. 74 (1981), no. 7, 3757-3773. MR 82d:81173

[17] D. Schmitt, B. Steffen, and T. Weiland, $2 D$ and $3 D$ computations of lossy eigenvalue problems, IEEE Trans. Magnetics 30 (1994), no. 5, 3578-3581.

[18] Steven M. Serbin, On factoring a class of complex symmetric matrices without pivoting, Math. Comp. 35 (1980), no. 152, 1231-1234. MR 81i:65025

[19] Robert J. Vanderbei, Symmetric quasi-definite matrices, SIAM J. Optimization 5 (1995), no. 1, 100-113. MR 95m:65078

[20] H. A. Van Der Vorst and J. B. M. Melissen, A Petrov-Galerkin type method for solving $A x=b$, where $A$ is symmetric complex, IEEE Trans. Magnetics 26 (1990), no. 2, 706-708.

Department of Mathematics, University of Manchester, Manchester, M13 9PL, EngLAND

E-mail address: higham@ma.man.ac.uk

URL: http://www.ma.man.ac.uk/ higham/ 